\title{
AN EXISTENCE RESULT FOR A SEMIPOSITONE PROBLEM WITH A SIGN CHANGING WEIGHT
}

\author{
JAFFAR ALI AND R. SHIVAJI
}

Received 5 March 2005; Accepted 5 September 2005

We establish an existence result on positive solution for a class of reaction-diffusion equation with semipositone structure. In particular, our results apply to the diffusive logistic equation with a class of sign changing weight and constant yield harvesting. We establish the result via the method of subsuper solutions.

Copyright (C) 2006 J. Ali and R. Shivaji. This is an open access article distributed under the Creative Commons Attribution License, which permits unrestricted use, distribution, and reproduction in any medium, provided the original work is properly cited.

\section{Introduction}

In this paper we discuss the existence of positive classical solutions $\left(u \in C^{2, \alpha}(\bar{\Omega})\right)$ of the boundary value problem

$$
\begin{gathered}
-\Delta u=\lambda\left(g(x)\left[u\left(1-u^{p}\right)\right]-\operatorname{ch}(x)\right), \quad x \in \Omega, \\
u=0, \quad x \in \partial \Omega,
\end{gathered}
$$

where $p>0, c>0$, and $\lambda>0$ are parameters and $\Omega$ is an open bounded region with boundary $\partial \Omega$ in class $C^{2}$ in $\mathbb{R}^{n}$ for $n \geq 1$. Here $g: \bar{\Omega} \rightarrow \mathbb{R}$ is a $C^{\alpha}$ function while $h: \Omega \rightarrow$ $\mathbb{R}$ is a nonnegative $C^{\alpha}$ function with $\|h\|_{\infty}=1$. When $p=1,(1.1)$ arises in population dynamics where $1 / \lambda$ is the diffusion coefficient and $\operatorname{ch}(x)$ represents the constant yield harvesting. In this case $(p=1)$, when $g(x)$ is a positive constant, various results have been established in [4]. Here we focus on sign changing weight functions $g$.

To precisely define our classes of weight functions, we first let $\lambda_{1}>0$ be the principal eigenvalue and $\phi>0$ with $\|\phi\|_{\infty}=1$ the corresponding eigenfunction of $-\Delta$ with the Dirichlet boundary conditions. It is well known that $\partial \phi / \partial \eta<0$ on $\partial \Omega$ where $\eta$ is the unit outward normal. Hence there exists $\delta>0, \sigma>0$, and $m>0$ such that

$$
\begin{gathered}
|\nabla \phi|^{2}-\lambda_{1} \phi^{2} \geq m \quad \text { on } \bar{\Omega}_{\delta}, \\
\phi \geq \sigma \quad \text { on } \Omega-\bar{\Omega}_{\delta},
\end{gathered}
$$

where $\Omega_{\delta}:=\{x \in \Omega \mid d(x, \partial \Omega)<\delta\}$. 
2 A semipositone problem with a sign changing weight

In this paper we assume that the weight $g$ takes negative values in $\Omega_{\delta}$ but requires $g$ to be strictly positive in $\Omega-\Omega_{\delta}$. Define $\gamma:=\min _{\Omega-\Omega_{\delta}} g(x), \mu:=\min _{\Omega_{\delta}} g(x)$, and we assume that

$$
|\mu|<\frac{m \gamma}{\lambda_{1}}\left(\frac{1}{p+1}\right)^{1 / p}
$$

Further let $0<x_{1}<x_{2}<\gamma / 2 \lambda_{1}$ be the positive roots of $q(x)=-\mu$ (see Figure 1.1), where

$$
q(x):=x\left[1-\frac{2 \lambda_{1}}{\gamma} x\right]^{1 / p}\left(\frac{p+1}{p}\right) 2 m .
$$

Then we establish the following.

Theorem 1.1. Suppose (1.4) holds, $1 / x_{2}<\lambda<1 / x_{1}$ and $c \leq c_{0}(\lambda)$, where

$c_{0}(\lambda):=\min \left\{\left(\frac{1}{p+1}\right)^{1 / p}\left[\frac{2 m}{\lambda}\left(1-\frac{2 \lambda_{1}}{\lambda \gamma}\right)^{1 / p}+\frac{\mu p}{(p+1)}\right], \frac{p \gamma \sigma^{2}}{(p+1)^{(p+1) / p}}\left[1-\frac{2 \lambda_{1}}{\lambda \gamma}\right]^{(p+1) / p}\right\}$.

Then (1.1) has at least one positive solution $u$ such that $\|u\|_{\infty}<1$.

Note that when $c>0,(1.1)$ is a semipositone problem and it is well known in the literature that the study of positive solutions is mathematically challenging (see [2-4]). Here we also include the additional challenge of dealing with a sign changing weight function $g$.

Finally, we also deduce a result for the case when $g(x) \geq 0$ on $\bar{\Omega}_{\delta}$. In particular we prove the following.

Corollary 1.2. If $g(x) \geq 0$ on $\bar{\Omega}_{\delta}$ and $c=0$, then for any $\lambda \geq 2 \lambda_{1} / \gamma$ (1.1) has a positive solution.

We establish our results by the method of subsuper solutions. By a subsolution we mean a function $w \in C^{2}(\bar{\Omega})$ such that

$$
\begin{gathered}
-\Delta w \leq \lambda\left(g(x)\left[w\left(1-w^{p}\right)\right]-\operatorname{ch}(x)\right), \quad x \in \Omega, \\
w \leq 0, \quad x \in \partial \Omega,
\end{gathered}
$$

and by a supersolution a function $v \in C^{2}(\bar{\Omega})$ such that

$$
\begin{gathered}
-\Delta v \geq \lambda\left(g(x)\left[v\left(1-v^{p}\right)\right]-\operatorname{ch}(x)\right), \quad x \in \Omega, \\
v \geq 0, \quad x \in \partial \Omega .
\end{gathered}
$$

Then it is well known (see $[1,5])$ that if there exists a subsolution $w$ and a supersolution $v$ such that $w<v$, then there exists a solution $u \in C^{2}(\bar{\Omega})$ such that $w \leq u \leq v$.

We will prove Theorem 1.1 in Section 2 and Corollary 1.2 in Section 3. 


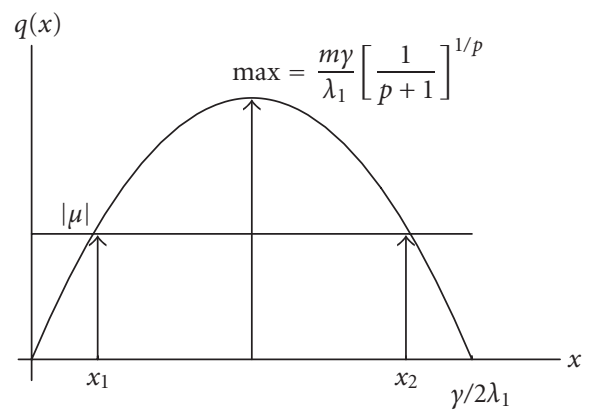

Figure 1.1

\section{Proof of Theorem 1.1}

Proof. Let $w=k_{0} \phi^{2}$, where

$$
k_{0}=\left(\frac{1}{p+1}\right)^{1 / p}\left[1-\frac{2 \lambda_{1}}{\lambda \gamma}\right]^{1 / p}
$$

We will prove that $w$ is a subsolution. Now

$-\Delta w=-\nabla \cdot \nabla\left(k_{0} \phi^{2}\right)=-\nabla \cdot\left(2 k_{0} \phi \nabla \phi\right)=-2 k_{0}(\nabla \phi \cdot \nabla \phi+\phi \Delta \phi)=2 k_{0}\left(\lambda_{1} \phi^{2}-|\nabla \phi|^{2}\right)$.

First we consider the case when $x \in \bar{\Omega}_{\delta}$. Since the maximum of $s\left(1-s^{p}\right)$ is $p /(p+$ $1)^{(p+1) / p}$, we have

$$
\lambda\left(g(x)\left[w\left(1-w^{p}\right)\right]-\operatorname{ch}(x)\right) \geq \lambda\left(\mu\left[\frac{p}{(p+1)^{(p+1) / p}}\right]-c\right) .
$$

Since

$$
c<c_{0} \leq\left(\frac{1}{p+1}\right)^{1 / p}\left[\frac{2 m}{\lambda}\left(1-\frac{2 \lambda_{1}}{\lambda \gamma}\right)^{1 / p}+\frac{\mu p}{(p+1)}\right]=\frac{2 k_{0} m}{\lambda}+\frac{\mu p}{(p+1)^{(p+1) / p}}
$$

combining (2.3)-(2.4) and using (1.2)-(2.2), we have

$$
\lambda\left(\mu\left[\frac{p}{(p+1)^{(p+1) / p}}\right]-c\right) \geq-\Delta w .
$$

Hence

$$
-\Delta w \leq\left(g(x)\left[w\left(1-w^{p}\right)\right]-\operatorname{ch}(x)\right) \quad \text { on } \bar{\Omega}_{\delta} .
$$


4 A semipositone problem with a sign changing weight

Next consider the case when $x \in \Omega-\bar{\Omega}_{\delta}$. By the definition of $\gamma$, we have

$$
\begin{aligned}
\lambda(g(x) & {\left.\left[w\left(1-w^{p}\right)\right]-\operatorname{ch}(x)\right) } \\
& \geq \lambda\left(\gamma\left[k_{0} \phi^{2}\left(1-k_{0}^{p} \phi^{2 p}\right)\right]-c\right) \geq \lambda\left(\gamma\left[k_{0} \phi^{2}\left(1-k_{0}^{p}\right)\right]-c\right) \\
& \geq \lambda\left(\gamma\left[k_{0} \phi^{2}\left(1-k_{0}^{p}\right)\right]-\frac{p \gamma}{(p+1)^{(p+1) / p}}\left[1-\frac{2 \lambda_{1}}{\lambda \gamma}\right]^{(p+1) / p} \sigma^{2}\right) \quad \text { since } c \leq c_{0} \\
& \geq \lambda\left(\gamma\left[k_{0} \phi^{2}\left(1-k_{0}^{p}\right)\right]-\frac{p \gamma}{(p+1)}\left[1-\frac{2 \lambda_{1}}{\lambda \gamma}\right] k_{0} \phi^{2}\right) \quad \text { using }(1.3),(2.1) \\
& =\lambda \gamma k_{0} \phi^{2}\left\{1-k_{0}^{p}-\frac{p}{(p+1)}\left[1-\frac{2 \lambda_{1}}{\lambda \gamma}\right]\right\} \\
& =\lambda \gamma k_{0} \phi^{2}\left\{1-k_{0}^{p}-p k_{0}^{p}\right\} \quad \text { by }(2.1) \\
& =\lambda \gamma k_{0} \phi^{2}\left\{1-[p+1] k_{0}^{p}\right\} \quad \text { by }(2.1) \\
= & \lambda \gamma k_{0} \phi^{2}\left\{1-\left[1-\frac{2 \lambda_{1}}{\lambda \gamma}\right]\right\} \quad \text { using }(2.2) . \\
= & 2 k_{0} \lambda_{1} \phi^{2} \geq 2 k_{0}\left[\lambda_{1} \phi^{2}-|\nabla \phi|^{2}\right] \\
= & -\Delta w \quad
\end{aligned}
$$

Hence

$$
-\Delta w \leq\left(g(x)\left[w\left(1-w^{p}\right)\right]-\operatorname{ch}(x)\right) \quad \text { on } \Omega-\bar{\Omega}_{\delta} .
$$

From (2.6) and (2.8) we have

$$
-\Delta w \leq\left(g(x)\left[w\left(1-w^{p}\right)\right]-\operatorname{ch}(x)\right) \quad \text { on } \Omega .
$$

Thus $w=k_{0} \phi^{2}$ is a subsolution of (1.1).

Next it is easy to see that $v \equiv 1$ is a supersolution of (1.1) and $v>w$ on $\bar{\Omega}$. Thus we have a positive solution $u$ such that $\|u\|_{\infty}<1$.

\section{Proof of Corollary 1.2}

Proof. Since $g(x) \geq 0$ and $c=0$, on $\bar{\Omega}_{\delta}, \lambda\left(g(x)\left[w\left(1-w^{p}\right)\right]\right) \geq 0$. But $-\Delta w \leq-2 k_{0} m$ and is negative; hence, on $\bar{\Omega}_{\delta}$, we have

$$
-\Delta w \leq g(x)\left[w\left(1-w^{p}\right)\right] \quad \text { on } \bar{\Omega}_{\delta}
$$


and on $\Omega-\bar{\Omega}_{\delta}$, we have

$$
\begin{aligned}
\lambda g(x) & {\left[w\left(1-w^{p}\right)\right] } \\
& \geq \lambda \gamma\left[k_{0} \phi^{2}\left(1-k_{0}^{p} \phi^{2 p}\right)\right] \geq \lambda \gamma\left[k_{0} \phi^{2}\left(1-k_{0}^{p}\right)\right] \\
& \geq \lambda \gamma k_{0} \phi^{2}\left[1-\frac{1}{p+1}\left[1-\frac{2 \lambda_{1}}{\lambda \gamma}\right]\right] \quad \text { by }(2.1) \\
& =\frac{k_{0} \phi^{2}}{p+1}\left[p \lambda \gamma+2 \lambda_{1}\right] \\
& \geq \frac{k_{0} \phi^{2}}{p+1}\left[2 \lambda_{1}(p+1)\right] \quad \text { since } \lambda \geq \frac{2 \lambda_{1}}{\gamma} \\
& =2 \lambda_{1} k_{0} \phi^{2} \\
& \geq 2 k_{0}\left[\lambda_{1} \phi^{2}-|\nabla \phi|^{2}\right]=-\Delta w .
\end{aligned}
$$

Hence we have

$$
-\Delta w \leq g(x)\left[w\left(1-w^{p}\right)\right] \quad \text { on } \Omega-\bar{\Omega}_{\delta}
$$

Using (3.1)-(3.3) we have that $w=k_{0} \phi^{2}$ is a subsolution. Again we note that $v \equiv 1$ is a supersolution. Hence the result holds.

\section{References}

[1] H. Amann, Fixed point equations and nonlinear eigenvalue problems in ordered Banach spaces, SIAM Review 18 (1976), no. 4, 620-709.

[2] H. Berestycki, L. A. Caffarelli, and L. Nirenberg, Inequalities for second-order elliptic equations with applications to unbounded domains. I, Duke Mathematical Journal 81 (1996), no. 2, 467494, A celebration of John F. Nash Jr.

[3] P. L. Lions, On the existence of positive solutions of semilinear elliptic equations, SIAM Review. A Publication of the Society for Industrial and Applied Mathematics 24 (1982), no. 4, 441-467.

[4] S. Oruganti, J. Shi, and R. Shivaji, Diffusive logistic equation with constant yield harvesting. I. Steady states, Transactions of the American Mathematical Society 354 (2002), no. 9, 3601-3619.

[5] D. H. Sattinger, Monotone methods in nonlinear elliptic and parabolic boundary value problems, Indiana University Mathematics Journal 21 (1971/1972), 979-1000.

Jaffar Ali: Department of Mathematics, Mississippi State University, Mississippi State, MS 39762, USA

E-mail address: js415@ra.msstate.edu

R. Shivaji: Department of Mathematics, Mississippi State University, Mississippi State, MS 39762, USA

E-mail address: shivaji@ra.msstate.edu 


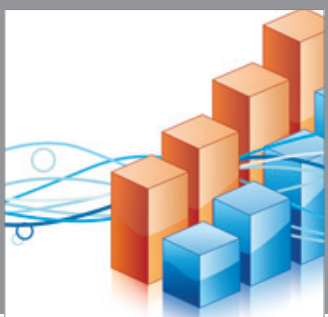

Advances in

Operations Research

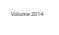

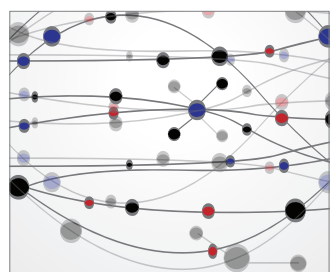

\section{The Scientific} World Journal
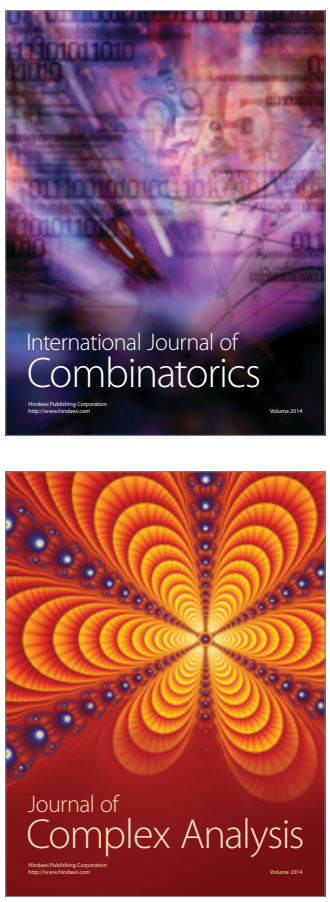

International Journal of

Mathematics and

Mathematical

Sciences
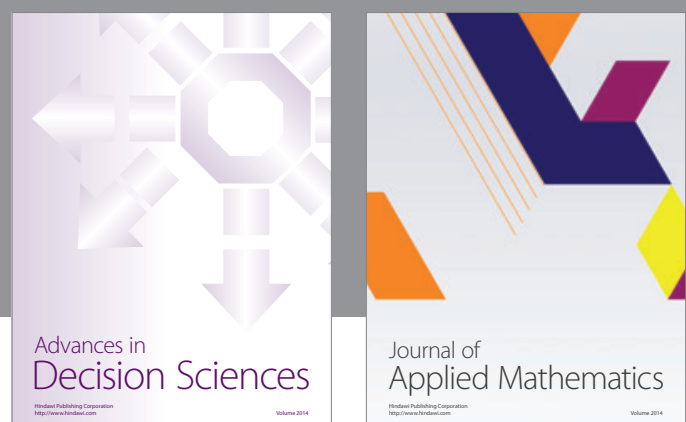

Journal of

Applied Mathematics
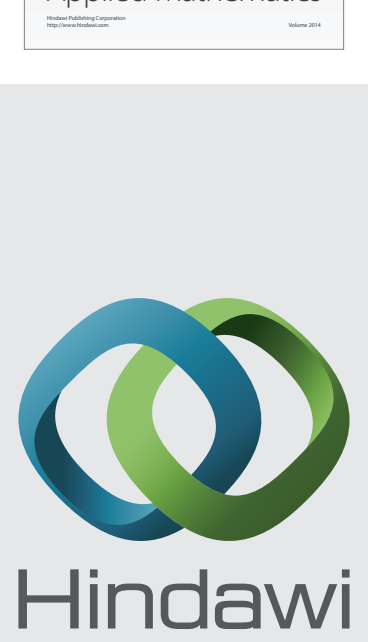

Submit your manuscripts at http://www.hindawi.com
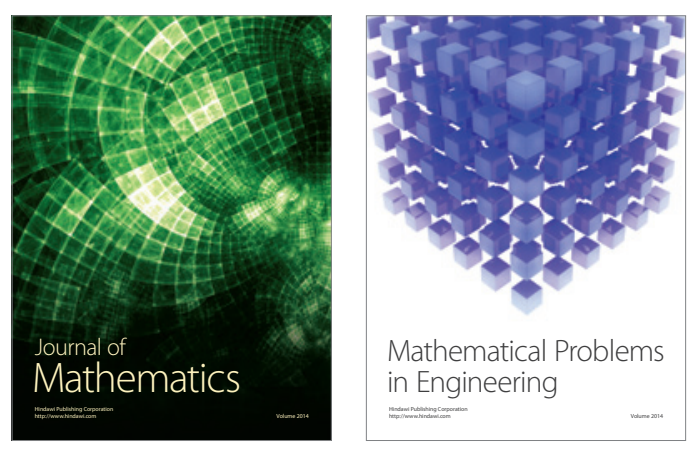

Mathematical Problems in Engineering
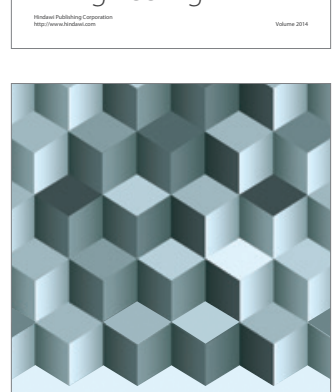

Journal of

Function Spaces
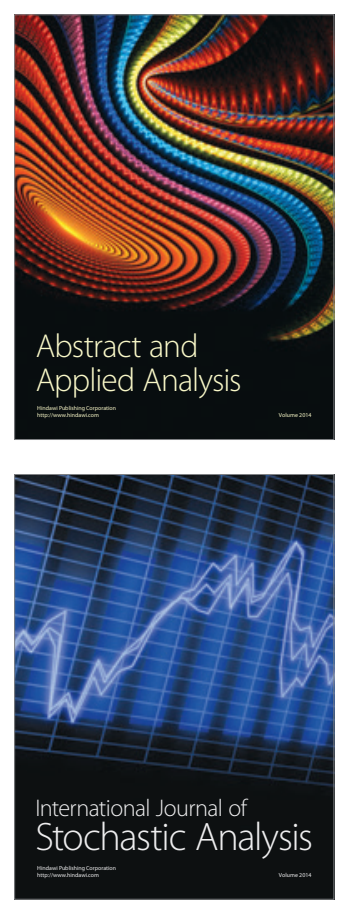

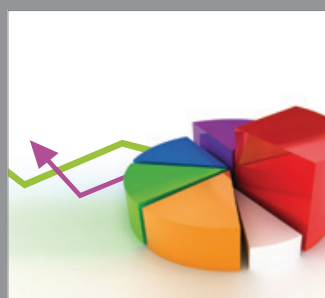

ournal of

Probability and Statistics

Promensencen
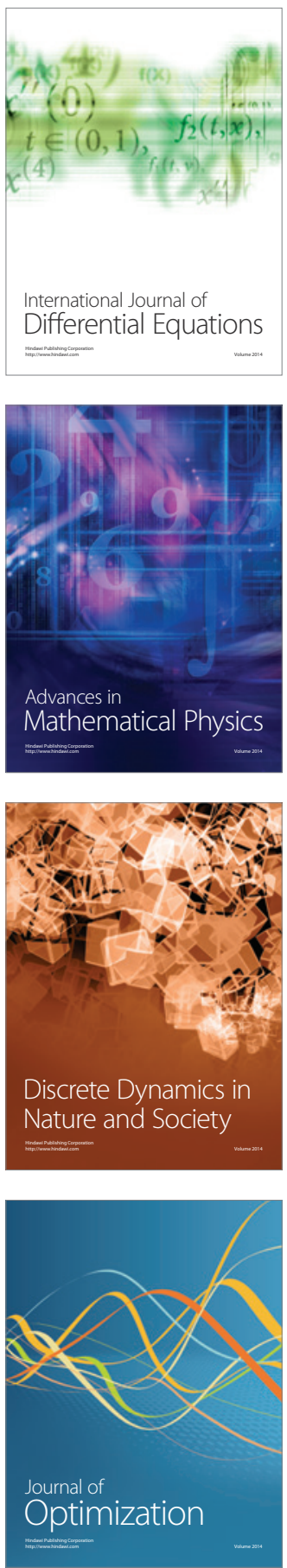